\title{
Special Features on Intelligent Imaging and Analysis
}

\author{
Dosik Hwang * and DaeEun Kim * (it) \\ School of Electrical and Electronic Engineering, Yonsei University, Seoul 03722, Korea \\ * Correspondence: dosik.hwang@yonsei.ac.kr (D.H.); daeeun@yonsei.ac.kr (D.K.)
}

Received: 29 October 2019; Accepted: 4 November 2019; Published: 10 November 2019

\section{Introduction}

Intelligent imaging and analysis have been studied in various research fields, including medical imaging, biomedical applications, computer vision, visual inspection and robot systems. Recently, artificial intelligence (AI) technologies with deep learning, machine learning and image processing have been applied to many difficult tasks, including challenging issues in image generation, reconstruction, de-noising, segmentation, and defect detection.

This Special Issue handles various applications of intelligent imaging technologies, covering medical imaging, visual detection, segmentation, medical diagnosis, image retrieval, image reconstruction and texture mapping. It shows the trend and the latest developments of intelligent imaging and analysis techniques. Theories of image processing, learning schemes and state-of-art artificial intelligence techniques for imaging and analysis are introduced.

\section{Intelligent Imaging and Analysis}

This section provides overall summaries of the papers included in this Special Issue for quick guidance for readers. The first half introduces non-medical or general applications, and the second half focuses on medical applications of the intelligent imaging and analysis techniques.

As an application of image analysis, face sketch synthesis has been tested by Wan and Lee [1], using the joint training method on face photos and sketches. Thus, more detailed information can be recorded in the synthesized sketches. Autonomous underwater vehicles (AUVs) have been a challenging subject requiring the underwater location accuracy. The work suggested by Wang et al. [2] shows the PL-SLAM (point and line simultaneous localization and mapping) to improve the accuracy of localization in the underwater environment. To measure volumetric tooth wear, a set of sequence images over sprocket teeth in a scraper conveyer can be collected. A focused morphology restoration algorithm has been applied to the image set by Ding et al. [3]. The method uses image filtering with a normal distribution operator to improve the accuracy of an evaluation function. Statistical body shape models can be used to estimate 3D human pose. However, self-intersection often occurs on images with a pattern of poses, especially between body parts. Wu et al. [4] introduced a self-intersection penalty term for statistical body shape models for 3D pose estimation, thus improving the accuracy of the pose estimation.

Many imaging techniques have been recently developed with CNN (convolutional neural network). A new approach with a depth module has been applied to human parsing by Jiang and Chi [5]. The method integrates a depth estimation module and a segmentation module as a variation of CNN (convolutional neural network) for image analysis, thus improving the performance for human parsing. Another CNN approach was used for scene parsing in the road scene context by Li et al. [6]. They provided a fast 3D semantic mapping system with monocular vision by combining localization, mapping and scene parsing. The semantic segmentation runs on selected key frames and their depth information, reducing the computational cost and also improving the accuracy of semantic mapping. 
The CNN model was also applied to geological structure image classification. As Zhang et al. [7] showed, transfer learning based on a deep learning model is effective to extract features of geological structure data. Oil spill detection on the ocean surface has been a hot issue, and a refined technique is required to detect dark spots on SAR (synthetic aperture radar) images. A deep convolution neural network, called Segnet has been tested for this application by Guo et al. [8]. It has the basic framework of encoder and decoder for image semantic segmentation. It improves accuracy performance to extract an oil spill location and area.

Three-dimensional image modeling reconstructs the 3D model of an object with multiple 2D images as well as maintaining its texture. Lai et al. [9] proposed a texture mapping method to use mesh partitioning, mesh parameterization and packing, texture transferring, and texture correction and optimization. It forms a high-resolution texture map of a 3D model for application in e-commerce. Image super-resolution technique is one of the most promising CNN based image processing techniques, which can be applied to various kinds of images. Chen et al. [10] propose a dual-channel CNN super-resolution network to extract the detailed texture information (deep channel) and the overall outline of the original image (shallow channel). Image quality assessment is an important process to maintain good quality of images for various types of imaging system. Jang et al. [11] proposed a new method of automatically assessing image quality when several enhancement techniques are applied, by using feature sets derived from high dynamic range images.

Detection-specific AI techniques such as the region-based CNN (R-CNN) can be used for various industrial purposes with specific configuration of a vision system. Wang et al. [12] proposed a novel one-camera-five-mirror system for cavitation bubble cluster study and have used a faster R-CNN method to detect bubbles in their system. The concept of residual network has widely been adopted in various deep learning architectures. Guo et al. [13] proposed an efficient deep residual network approach by using sparse feedbacks, which improves the convergence speed and the training stability in their image restoration applications.

Image segmentation has been tackled with a novel active contour model by Sun et al. [14]. It uses an improved SPF (signed pressure function) and a LIF (local image fitting) model. A weight function of the grayscale mean values around the contour curve was introduced to segment blurred images and weak gradient images, and another metric function was used to check local image information to segment intensity-inhomogeneous images. Image segmentation plays a role of monitoring the weld pool surface affecting the weld quality. The reflected laser lines used for arc welding contain the weld pool surface information, and Wang et al. [15] proposed that various image processing methods are combined for image segmentation approach, applied to the reflected laser lines. An effective image search is demanding work in the field of content-based image retrieval. A novel approach to encode the relative spatial information for histogram-based representation of the visual worlds has been introduced by Zafar et al. [16]. It computes the geometric relationship in the visual worlds and enhances the performance of image retrieval.

Surface defect detection is a challenging problem in industrial product manufacturing. Vision-based defect detection of steel sheets focuses on finding the salient characteristics of the defects. Zhou et al. [17] processed the defect segmentation with a double low-rank and sparse decomposition model to obtain high-quality defect images. Another style of surface defect detection can be found in the railway surface. A novel visual inspection approach based on UAV (unmanned aerial vehicle) images has been tested by Wu et al. [18]. It characterizes the defective sub-regions and defect-free background sub-regions, and highlights the critical defect regions in the image analysis. Their approach has two key techniques for UAV-based rail images: image enhancement and surface defect segmentation.

The inspection of a PCB (printed circuit board) is a kind of surface defect detection problem with visual image in manufacturing. Yuk et al. [19] extracted robust features in the visual image with various types of defect patterns such as scratches and improper etching, applying a random forest method. They also used probabilistic kernel density estimation to improve the detection performance. 
Visual inspection technology in the manufacturing process in the iron and steel industry has been investigated by Sun et al. [20]. Coverage of cameras or light source information can differentiate the hardware selection of visual inspection. The inspection or detection algorithm depends on filtering, statistics and learning methods. Basic theories and foundations of image processing highly contribute to the visual inspection technologies, according to this review paper.

The following part introduces medical applications of intelligent imaging and analysis techniques on various types of image datasets including magnetic resonance images (MRI), computed tomography (CT), optical scan data, photographic images and digital videos.

Segmentation of medical images is one of the most important tasks for many medical image analyses. However, the boundaries of the object segmentation have not been well addressed so far. Kim et al. [21] proposed a method to overcome the poor performance issues encountered on the boundaries of objects. Their boundary-specific U-net improved the segmentation performance on the boundaries of the intervertebral discs in MR spine images. Human lumbar spine diagnosis can be assisted by accurate segmentation of the vertebral bodies. Kim et al. [22] also proposed a semi-automatic segmentation technique for vertebral bodies in MR images which can reduce the user's role while achieving good segmentation accuracy.

A data balancing problem is an important issue to address for some deep learning applications, especially when there are not enough datasets for training. It is often noticed that non-balanced datasets lead to a biased estimation model. Zhang et al. [23] proposed a pre-training strategy to address this problem encountered in the liver segmentation from computed tomography (CT) scans. Zheng et al. proposed an intelligent evaluation system for strabismus diagnosis with a sequence of digital video analyses [24]. Each image in the recorded video is analyzed to localize important features, which are combined to evaluate the strabismus. Updating and automatic training can be an important step for the practical application and subsequence maintenance of an AI-based system. Sugimori [25] discussed the overall accuracy of additional learning and automatic training system for CT classification task.

Intelligent design and manufacturing can help medical procedures. Kim et al. [26] proposed a computer-aided design and manufacturing technology for patient-specific optimization of the Nuss procedure for minimally invasive surgery of pectus excavatum. This can be an example of how the intelligent imaging and analysis techniques can change the shape of the medical procedures in the future. Automatic alignment of images and volumes in medical imaging data is an important step for further subsequent analysis. Rehman and Lee [27] proposed an efficient automatic midsagittal plane extraction in brain MRI images. This method can be useful for image registration, asymmetric analysis and tilt correction encountered in the analysis of brain images.

Registration between volume data and surface data is also an important application in several medical procedures. Jung et al. [28] proposed an effective registration technique for dental tomographic volume data and scan surface data by using a dynamic segmentation technique. Another registration task can be found as a point cloud registration for 3D datasets. Liu et al. [29] presented a 3D point cloud registration algorithm based on a greedy projection triangulation method to address the $3 \mathrm{D}$ problem.

\section{Future Intelligent Imaging and Analysis}

Various technological advancements of intelligent imaging and analysis have been introduced in this Special Issue. With the advent of the deep learning and related machine learning techniques, many of the conventional imaging and analysis techniques are being improved or substituted by the intelligent learning-based methods. It is expected that robust and reliable intelligent techniques will soon be deployed for many practical applications in industries, sciences, medicine and arts.

Author Contributions: The authors of this editorial served as Guest Editors for this Special Issue. Both the authors contributed equally to this editorial and proofread for publication.

Funding: The first author was supported by Basic Science Research Program through the National Research Foundation of Korea (NRF) funded by the Ministry of Science and ICT (2019R1A2B5B01070488), Brain Research Program through NRF (2018M3C7A1024734), and Bio \& Medical Technology Development Program of NRF 
(NRF-2018M3A9H6081483). The second author was supported by the NRF funded by the Korea government (MSIT) (No. 2017R1A2B4011455).

Acknowledgments: We would like to thank all the authors who contributed to this Special Issue. Their contributions have made this Special Issue a very valuable one. We also thank all reviewers for their time and commitment to providing comments and suggestions. This issue would not be possible without their efforts and time. Finally, we would like to express our special thanks to the editorial team and Daria Shi, the managing editor of Applied Sciences for their kind and endless help to keep us focused on this Special Issue.

Conflicts of Interest: The authors declare no conflict of interest.

\section{References}

1. Wan, W.; Lee, H.J. A Joint Training Model for Face Sketch Synthesis. Appl. Sci. 2019, 9, 1731. [CrossRef]

2. Wang, R.; Wang, X.; Zhu, M.; Lin, Y. Application of a Real-Time Visualization Method of AUVs in Underwater Visual Localization. Appl. Sci. 2019, 9, 1428. [CrossRef]

3. Ding, H.; Liu, Y.; Liu, J. Volumetric Tooth Wear Measurement of Scraper Conveyor Sprocket Using Shape from Focus-Based Method. Appl. Sci. 2019, 9, 1084. [CrossRef]

4. Wu, Z.; Jiang, W.; Luo, H.; Cheng, L. A Novel Self-Intersection Penalty Term for Statistical Body Shape Models and Its Applications in 3D Pose Estimation. Appl. Sci. 2019, 9, 400. [CrossRef]

5. Jiang, Y.; Chi, Z. A CNN Model for Human Parsing Based on Capacity Optimization. Appl. Sci. 2019, 9, 1330. [CrossRef]

6. Li, X.; Wang, D.; Ao, H.; Belaroussi, R.; Gruyer, D. Fast 3D Semantic Mapping in Road Scenes. Appl. Sci. 2019, 9, 631. [CrossRef]

7. Zhang, Y.; Wang, G.; Li, M.; Han, S. Automated Classification Analysis of Geological Structures Based on Images Data and Deep Learning Model. Appl. Sci. 2018, 8, 2493. [CrossRef]

8. Guo, H.; Wei, G.; An, J. Dark Spot Detection in SAR Images of Oil Spill Using Segnet. Appl. Sci. 2018, 8, 2670. [CrossRef]

9. Lai, J.-Y.; Wu, T.-C.; Phothong, W.; Wang, D.W.; Liao, C.-Y.; Lee, J.-Y. A High-Resolution Texture Mapping Technique for 3D Textured Model. Appl. Sci. 2018, 8, 2228. [CrossRef]

10. Chen, Y.; Wang, J.; Chen, X.; Sangaiah, A.K.; Yang, K.; Cao, Z. Image Super-Resolution Algorithm Based on Dual-Channel Convolutional Neural Networks. Appl. Sci. 2019, 9, 2316. [CrossRef]

11. Jang, J.; Jang, H.; Eo, T.; Bang, K.; Hwang, D. No-reference Automatic Quality Assessment for Colorfulness-Adjusted, Contrast-Adjusted, and Sharpness-Adjusted Images Using High-Dynamic-Range-Derived Features. Appl. Sci. 2018, 8, 1688. [CrossRef]

12. Wang, H.; Xu, H.; Pooneeth, V.; Gao, X.-Z. A Novel One-Camera-Five-Mirror Three-Dimensional Imaging Method for Reconstructing the Cavitation Bubble Cluster in a Water Hydraulic Valve. Appl. Sci. 2018, 8, 1783. [CrossRef]

13. Guo, Z.; Sun, Y.; Jian, M.; Zhang, X. Deep Residual Network with Sparse Feedback for Image Restoration. Appl. Sci. 2018, 8, 2417. [CrossRef]

14. Sun, L.; Meng, X.; Xu, J.; Tian, Y. An Image Segmentation Method Using an Active Contour Model Based on Improved SPF and LIF. Appl. Sci. 2018, 8, 2576. [CrossRef]

15. Wang, Z.; Zhang, C.; Pan, Z.; Wang, Z.; Liu, L.; Qi, X.; Mao, S.; Pan, J. Image Segmentation Approaches for Weld Pool Monitoring during Robotic Arc Welding. Appl. Sci. 2018, 8, 2445. [CrossRef]

16. Zafar, B.; Ashraf, R.; Ali, N.; Iqbal, M.K.; Sajid, M.; Dar, S.H.; Ratyal, N.I. A Novel Discriminating and Relative Global Spatial Image Representation with Applications in CBIR. Appl. Sci. 2018, 8, 2242. [CrossRef]

17. Zhou, S.; Wu, S.; Liu, H.; Lu, Y.; Hu, N. Double Low-Rank and Sparse Decomposition for Surface Defect Segmentation of Steel Sheet. Appl. Sci. 2018, 8, 1628. [CrossRef]

18. Wu, Y.; Qin, Y.; Wang, Z.; Jia, L. A UAV-Based Visual Inspection Method for Rail Surface Defects. Appl. Sci. 2018, 8, 1028. [CrossRef]

19. Yuk, E.H.; Park, S.H.; Park, C.-S.; Baek, J.-G. Feature-Learning-Based Printed Circuit Board Inspection via Speeded-Up Robust Features and Random Forest. Appl. Sci. 2018, 8, 932. [CrossRef]

20. Sun, X.; Gu, J.; Tang, S.; Li, J. Research Progress of Visual Inspection Technology of Steel Products-A Review. Appl. Sci. 2018, 8, 2195. [CrossRef] 
21. Kim, S.; Bae, W.C.; Masuda, K.; Chung, C.B.; Hwang, D. Fine-Grain Segmentation of the Intervertebral Discs from MR Spine Images Using Deep Convolutional Neural Networks: BSU-Net. Appl. Sci. 2018, 8, 1656. [CrossRef] [PubMed]

22. Kim, S.; Bae, W.C.; Masuda, K.; Chung, C.B.; Hwang, D. Semi-Automatic Segmentation of Vertebral Bodies in MR Images of Human Lumbar Spines. Appl. Sci. 2018, 8, 1586. [CrossRef]

23. Zhang, Y.; Wang, Y.; Wang, Y.; Fang, B.; Yu, W.; Long, H.; Lei, H. Data Balancing Based on Pre-Training Strategy for Liver Segmentation from CT Scans. Appl. Sci. 2019, 9, 1825. [CrossRef]

24. Zheng, Y.; Fu, H.; Li, R.; Lo, W.-L.; Chi, Z.; Feng, D.D.; Song, Z.; Wen, D. Intelligent Evaluation of Strabismus in Videos Based on an Automated Cover Test. Appl. Sci. 2019, 9, 731. [CrossRef]

25. Sugimori, H. Evaluating the Overall Accuracy of Additional Learning and Automatic Classification System for CT Images. Appl. Sci. 2019, 9, 682. [CrossRef]

26. Kim, Y.-J.; Heo, J.-Y.; Hong, K.-H.; Lim, B.-Y.; Lee, C.-S. Computer-Aided Design and Manufacturing Technology for Identification of Optimal Nuss Procedure and Fabrication of Patient-Specific Nuss Bar for Minimally Invasive Surgery of PectusExcavatum. Appl. Sci. 2019, 9, 42. [CrossRef]

27. Rehman, H.Z.U.; Lee, S. An Efficient Automatic Midsagittal Plane Extraction in Brain MRI. Appl. Sci. 2018, 8, 2203. [CrossRef]

28. Jung, K.; Jung, S.; Hwang, I.; Kim, T.; Chang, M. Registration of Dental Tomographic Volume Data and Scan Surface Data Using Dynamic Segmentation. Appl. Sci. 2018, 8, 1762. [CrossRef]

29. Liu, J.; Bai, D.; Chen, L. 3-D Point Cloud Registration Algorithm Based on Greedy Projection Triangulation. Appl. Sci. 2018, 8, 1776. [CrossRef]

(C) 2019 by the authors. Licensee MDPI, Basel, Switzerland. This article is an open access article distributed under the terms and conditions of the Creative Commons Attribution (CC BY) license (http://creativecommons.org/licenses/by/4.0/). 